\title{
Time-delayed nonlocal response inducing traveling temporal localized structures
}

\author{
M. G. Clerc $\odot,{ }^{1}$ S. Coulibaly, ${ }^{2}$ and M. Tlidi $^{3}$ \\ ${ }^{1}$ Departamento de Física and Millennium Institute for Research in Optics, Facultad de Ciencias Físicas y Matemáticas, \\ Universidad de Chile, Casilla 487-3, Santiago, Chile \\ ${ }^{2}$ Université de Lille, CNRS, UMR 8523-PhLAM-Physique des Lasers Atomes et Molécules, F-59000 Lille, France \\ ${ }^{3}$ Département de Physique, Faculté des Sciences, Université Libre de Bruxelles (U.L.B.), CP 231, Campus Plaine, B-1050 Bruxelles, Belgium
}

(Received 13 December 2018; published 8 January 2020)

\begin{abstract}
We show analytically and numerically that time-delayed nonlocal response induces traveling localized states in bistable systems. These states result from the interaction of fronts between homogeneous steady states. We illustrate this mechanism by considering an experimentally relevant system - the fiber cavity with the noninstantaneous Raman response. Close to the nascent bistability, we performed a derivation of a generic bistable model with a nonlocal delayed response. Analytical expressions of the width and the speed of traveling localized states are derived. Without a time-delayed nonlocal response, traveling localized states are excluded. In addition, we propose realistic parameters and perform numerical simulations of the governing model equation.
\end{abstract}

DOI: 10.1103/PhysRevResearch.2.013024

\section{INTRODUCTION}

Macroscopic systems are regularly described by a small number of coarse-grained or macroscopic variables. The separation of timescales makes this reduction possible. Generally speaking, it allows for a description in terms of slowly varying macroscopic or averaging variables [1-3]. When scales separation of the micro- and macroscopic variables are not well established, the dynamics can be altered by the effect of temporal correlations that generate a temporal delayed response. A classic example is the polarization or the magnetization of a material subjected to external electric or magnetic fields. For low field strengths, at any given time, the polarization is $\mathbf{P}(\tau)=\int_{-\infty}^{\tau} \chi\left(\tau-\tau^{\prime}\right) \mathbf{E}\left(\tau^{\prime}\right) d \tau^{\prime}$, where $\mathbf{E}(t)$ is the electric field [4]. The electrical linear susceptibility $\chi(\tau)$ is the temporal delay kernel that accounts for the response of the charge density to the electrical stimulus. When the characteristic frequency of the electric field is much smaller than the frequency associated with the motion of the electric charges, the effect of time delay can reasonably be neglected, and the response of the system is instantaneous and local. However, the nonlocal response is the rule rather than the exception, and it is relevant for optical and magnetic systems such as scattering of light in a continuous medium [4], nonlinear optics [5], and fiber optics [6]. The time-delayed nonlocal response is a general physical phenomenon that is widely used also for biological systems [7-10].

In another line of research, frequency comb generation in microresonators has witnessed tremendous progress in recently, allowing new applications in metrology and spec-

Published by the American Physical Society under the terms of the Creative Commons Attribution 4.0 International license. Further distribution of this work must maintain attribution to the author(s) and the published article's title, journal citation, and DOI. troscopy [11]. Frequency combs generated in optical Kerr resonators are nothing but the spectral content of the stable temporal localized structure (LS) occurring in the cavity $[12,13]$. These peaks are generated close to the modulational instability. The link between the phenomenon of Kerr comb formation and temporal localized structures has been established (see the latest overview [14] in the theme issue [15]). In optical fibers, the nonlocal delayed response is provided by the Raman effect, which occurs spontaneously when an intense optical beam is passed through a fiber. It has been recently shown that the Raman effect may induce Kerr optical frequency comb generation [16]. So far, however, the formation of traveling localized structures induced by the time-delayed response, including the Raman effect, have neither been experimentally determined nor theoretically predicted. We address here the theoretical side of this problem in the regime devoid of any modulational instability.

We show that the nonlocal delay induces traveling localized states in an all-optical fiber cavity with a nonlocal delayed response, modeled by the Raman effect. We demonstrate numerically by using realistic parameters that this simple optical device supports traveling LS. From the generalized Lugiato-Lefever equation, we derive a generic bistable system. We provide an analytical understanding of the generation of traveling LS in terms of fronts interaction. We characterize these moving structures by deriving a coupled equation for the slow time evolution of their width and their speed. Numerical simulations show a fairly good agreement with the theoretical predictions.

The paper is organized as follows: In Sec. II, we present a generalized Lugiato-Lefever model describing all-fiber resonators with Raman delayed nonlocal response, and we show numerical evidence of moving localized structures. At the end of this section, we provide possible experimental parameter values relevant to the observation of traveling localized structures in an all-fiber cavity. In Sec. III, we present a summary of the derivation of a generic bistable equation with 
(a)
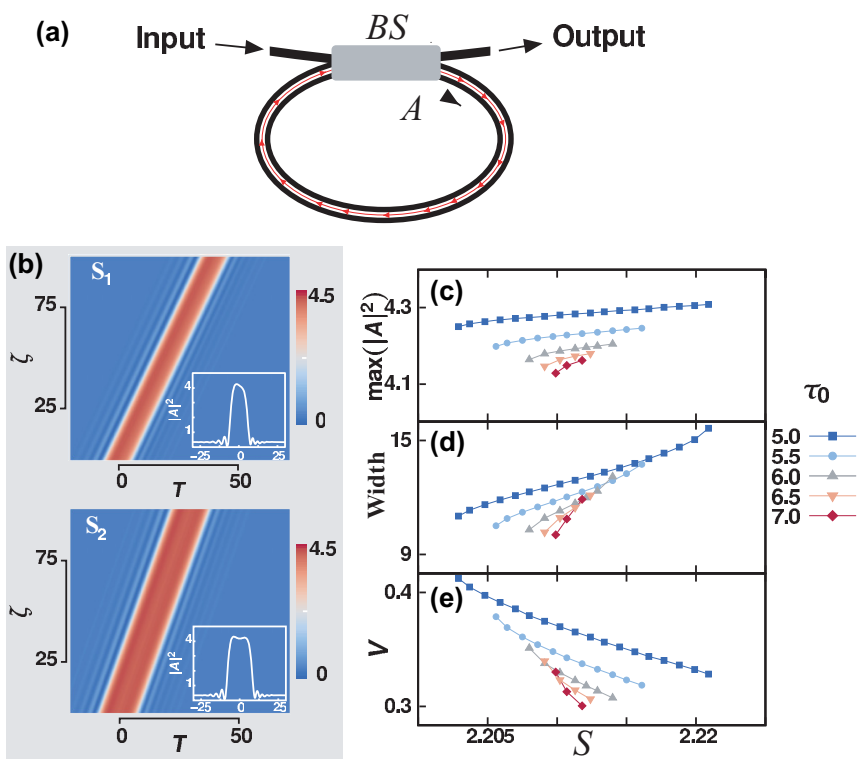

FIG. 1. Traveling localized structures in an all-fiber cavity with Raman response. (a) Schematic representation of an optical fiber ring cavity. BS accounts for the beam splitter. (b) $\zeta-T$ maps of traveling localized structures of Eq. (1) obtained for $\Delta=4, \tau_{0}=5 \mathrm{fs}, S_{1}=$ 2.202, $\left(S_{2}=2.223\right), f_{R}=0.18, \tau_{1}=12 \mathrm{fs}, b_{2}=1$, and $\tau_{2}=32 \mathrm{fs}$. (c) The maximum peak intensity, (d) the width, and (e) the speed of the traveling localized structures as a function of driven field amplitude as obtained for the same parameter settings as in (b) except for $S$ and $\tau_{0}$.

the delayed nonlocal response. This reduction is valid close to the critical point associated with bistability. In Sec. IV, we show analytically and numerically that delayed nonlocal response through front interaction allows for the stabilization moving localized structures. Finally, in Sec. V, we conclude.

\section{LUGIATO-LEFEVER EQUATION WITH RAMAN DELAYED NONLOCAL RESPONSE}

We consider all-fiber ring cavities coherently driven by an injected light beam as shown in Fig. 1(a). The envelope of the electric field within the cavity is described by the Lugiato-Lefever equation [17] with the Raman delayed nonlocal response [18]:

$$
\begin{aligned}
\frac{\partial A}{\partial \zeta}= & S-(1+i \Delta) A-i b_{2} \frac{\partial^{2} A}{\partial T^{2}}+i\left(1-f_{R}\right)|A|^{2} A \\
& +i f_{R} A \int_{-\infty}^{T} h_{R}^{\prime}\left(T-T^{\prime}\right)\left|A\left(T^{\prime}\right)\right|^{2} d T^{\prime} .
\end{aligned}
$$

The slow time describing the evolution over the successive round trips is $t=t_{R} \zeta / \kappa$. The time $\tau=\tau_{0} T$ with $\tau_{0}=$ $\sqrt{\left|\beta_{2} L\right| /(2 \kappa)}$ is the fast time in the reference frame moving with the group velocity of the light within the cavity. The injected field is $E_{i n}=\sqrt{\kappa^{3} /(\Gamma L \theta)} S, \theta$ effective transmission of the beam splitter, the intracavity field is $E(t, \tau)=$ $\sqrt{\kappa /(\Gamma L)} A(\zeta, T)$, and the normalized detuning is $\Delta=\delta / \kappa$. The losses, the phase detuning, the chromatic dispersion coefficient, the cavity length, the Kerr nonlinear coefficient, and the cavity round-trip time are denoted by $\kappa, \delta, \beta_{2}, L, \Gamma$, and $t_{R}$, respectively. The coefficient $b_{2}$ is positive assuming a normal dispersion regime $\left(b_{2}\right.$ accounts for the sign of the value of $\beta_{2}$, without loss of generality we can consider $b_{2}=$ 1 ), and it can be scaled down to unity. Finally, the Raman response is modeled by the function $h_{R}^{\prime}(\tau)=\tau_{0} h_{R}\left(\tau_{0} \tau\right)$, and $f_{R}$ measures the strength of the Raman response. In agreement with experiments, a more accurate form of this function has been proposed in Refs. [19,20]

$$
h_{R}(\tau)=\frac{\tau_{1}^{2}+\tau_{2}^{2}}{\tau_{1} \tau_{2}^{2}} e^{-\tau / \tau_{2}} \sin \left(\tau / \tau_{1}\right) .
$$

Observe that in the limit $\tau_{1} \rightarrow \infty$ the Raman response is of exponential nature $h_{R}(\tau) \propto e^{-\tau / \tau_{2}}$. In the absence of the Raman effect $\left(f_{R}=0\right)$, we recover, the well-known LugiatoLefever equation (LLE), which constitutes the paradigmatic model for the study of dissipative structures in nonlinear optics [17] (see also recent reports on that model, Refs. [14,21$23]$ and references therein). In the absence of Raman effect, i.e., $f_{R}=0$, fronts [24], motionless LS connecting homogeneous solutions [25], moving LS due to the third-order dispersion effect [26-28], spatiotemporal chaos [29,30], and rogue waves $[31,32]$ have been reported.

In spatially extended systems with local coupling such as diffraction, the motion of localized structures is triggered by a phase gradient [33-37], thermal effects [38-40], walk-off [24,41], Ising-Bloch transition [42-44], frequency-selective $[45,46]$ feedback, or regular delayed feedback [47-50]. The spontaneous motion of LS has been reported in lasers with a saturable absorber [51-55].

Examples of traveling LS obtained from numerical simulations of Eq. (1) are shown in Fig. 1(b). These structures are asymmetric nonlinear objects with a pronounced oscillatory tail [cf. the insets of Fig. 1(b)]. The characteristics of these solutions such as width, maximum peak intensity, and speed are strongly affected by the driving field amplitude and by the group velocity dispersion through the characteristic time $\tau_{0}$, as shown in Figs. 1(c)-1(d). From these figures, we see that the existence domain of moving LS increased with $\tau_{0}$, and hence with the group velocity dispersion. The maximum peak intensity, as well as the width of moving LS, increased with the injected field amplitude as shown in Figs. 1(c) and 1(d). However, when increasing the injected field amplitude, the speed of traveling solutions decreases as shown in Fig. 1(e). We now provide possible experimental parameter values relevant to the observation of traveling LS in all-fiber cavities. The fiber ring cavity physical parameters are $L=10 \mathrm{~m}, \beta_{2} \in$ $\left[10^{-6} ; 1.8 \times 10^{-6}\right] \mathrm{ps}^{2} / \mathrm{m}, \kappa=0.26, \Gamma=0.01(\mathrm{~W} \mathrm{~m})^{-1}, \delta=$ $1.04, f_{R}=0.18, \tau_{1}=12 \mathrm{fs}$, and $\tau_{2}=32 \mathrm{fs}$.

\section{DERIVATION OF A BISTABLE EQUATION WITH RAMAN DELAYED NONLOCAL RESPONSE}

The homogeneous steady-state solutions of Eq. (1) are $S^{2}=\left|A_{s}\right|^{2}\left[1+\left(\Delta-\left|A_{s}\right|^{2}\right)^{2}\right]$. These states remain unaltered by the Raman effect. To show analytically and numerically that nonlocal delayed response stabilizes traveling LS solutions of Eq. (1), we restrict our analysis close to a secondorder critical point marking the onset of a hysteresis loop where the inflection point (with infinite slope) of the intensity response curve corresponds to the critical detuning for 
the onset of optical bistability given by $\Delta=\Delta_{c} \equiv \sqrt{3}$. At this critical point, the coordinate of the intracavity field is $A_{c}=(3-i \sqrt{3}) S_{c} / 4=A_{r c}+i A_{i c}$, where $S_{c}^{2}=8 \sqrt{3} / 9$ is the value of the injected field at criticality. We introduce a small parameter $\epsilon$ that measures the distance from the critical point as $\Delta=\Delta_{c}\left(1+\delta \epsilon^{2}\right)$. We expand the injected field in terms of $\epsilon$ as $S=S_{c}\left[1+3 \delta \epsilon^{2} / 4+s \epsilon^{3}\right]$. Then we decompose the electric field into its real and imaginary parts as $A=A_{r}+$ $i A_{i}$ and we expand $A_{r}=A_{r c}\left(1+\epsilon u+\epsilon^{2} u_{1}+\cdots\right)$ and $A_{i}=$ $A_{i c}\left(1+\epsilon v_{0}+\epsilon^{2} v_{1}+\cdots\right)$. We focus on the situation where the strength of the Raman effect is weak, i.e., $f_{R}=\epsilon^{2} f$, and seek corrections to the steady states at criticality that depend on time and space through the slow variables $t=\epsilon^{2} \zeta$ and $\tau=3^{1 / 4} \epsilon T$. The higher-order inhomogeneous problem yields the generic bistable system with a delayed nonlocal response (see the Appendix):

$$
\partial_{t} u=\eta+\mu u-u^{3}+D \partial_{\tau \tau} u+\int_{-\infty}^{\tau} \chi\left(\tau-\tau^{\prime}\right) u\left(\tau^{\prime}\right) d \tau^{\prime},
$$

where $u=u(t, \tau)$ is a scalar order parameter. The parameters $\eta, \mu, D$, and $t$ are $t \rightarrow 2 t / 3, \eta=2 s, \mu=(3 \delta-2 f) / 2$, and $D=3 b_{2} / 2$. The delay kernel function $\chi(\tau)$ is

$$
\chi(\tau)=\frac{2 a f}{3} e^{\frac{-\tau_{0} \tau}{\tau_{2}}} \sin \left(\tau_{0} \tau / \tau_{1}\right),
$$

with $a=\tau_{0}\left(\tau_{1}^{2}+\tau_{2}^{2}\right) / \tau_{1} \tau_{2}^{2}$. In the limit of large $\tau_{1}$ and $\tau$, Eq. (3) can approximated by

$$
\partial_{t} u=\eta+\mu u-u^{3}+D \partial_{\tau \tau} u+\frac{\gamma}{\alpha} \int_{-\infty}^{\tau} e^{-\alpha\left(\tau-\tau^{\prime}\right)} u\left(\tau^{\prime}\right) d \tau^{\prime},
$$

where $\alpha=\tau_{0} / \tau_{2}$ and $\gamma=2 \tau_{1} f /\left(3 \tau_{2}\right)$ account for the characteristic correlation time and the strength of the nonlocal delayed response, respectively. The comparison in terms of the width and the speed of moving localized structures is provided in the Appendix. The results obtained from Eqs. (3) and (5) are excellent when $\tau_{1}$ becomes large, as shown in the Appendix. In the absence of nonlocal delay, i.e, $\gamma=0$, the above real order parameter resulting from a multiscale analysis has been compared with the LLE theoretically [28] and experimentally [56]. Equation (5) can be written as $\partial_{t} u=$ $-\partial_{u} V(u)+D \partial_{\tau \tau} u$, with $V(u)=-\eta u-\mu u^{2} / 2+u^{4} / 4$. For $\eta^{2}<\mu^{3} / 9$, the potential $V(u)$ possesses two symmetric front solutions which are motionless only at the Maxwell point defined by $\eta=0$, as shown by the dashed curve of Fig. 2(a). The motionless front connecting the two symmetric states, $u_{0}= \pm \sqrt{\mu}$, is plotted in Fig. 2(b). For $\gamma=0$, and at the Maxwell point, Eq. (5) admits an exact nonlinear front solution given by $u_{ \pm}(\tau)= \pm \sqrt{\mu} \tanh \left[\sqrt{\mu / 2}\left(\tau-\tau_{p}\right)\right]$, with $\tau_{p} \equiv$ $\int_{-\infty}^{\infty} \tau \partial_{\tau} u_{ \pm}(\tau) d \tau / \int_{-\infty}^{\infty} \partial_{\tau} u_{ \pm}(\tau) d \tau$ as the front position. Far from the Maxwell point $\eta \neq 0$ [solid line in Fig. 2(a)], the front exhibits a motion with a constant speed as shown in Fig. 2(c).

\section{TIME-DELAYED NONLOCAL RESPONSE INDUCES TRAVELING LOCALIZED STATES: INTERACTION BETWEEN FRONTS}

When two opposite fronts are at some distance from each other, they interact in an attractive or repulsive way depending
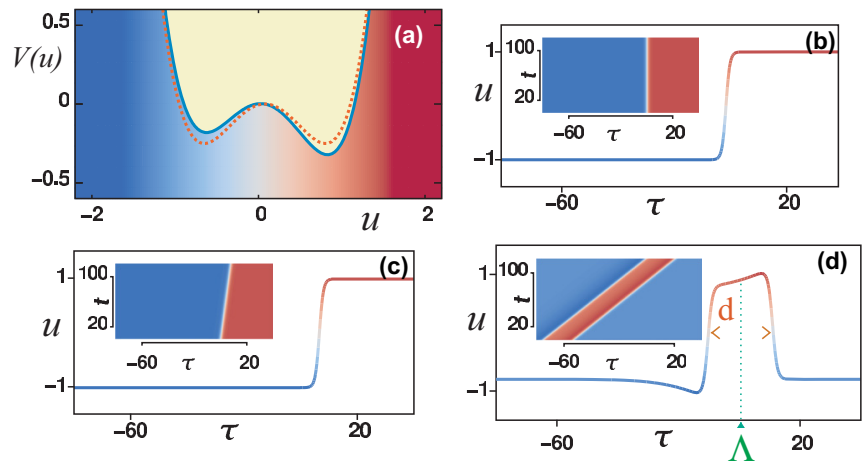

FIG. 2. Fronts and traveling localized structure of Eq. (5). (a) The potential $V(u)$ obtained for $\gamma=0, \mu=1, \eta=0$ (dashed line), and $\eta=-0.03$ (solid line). (b) The front solution at the Maxwell point. Parameters are $\gamma=0, \mu=1$, and $\eta=0$. (c) The front propagation obtained for $\gamma=0, \mu=1$, and $\eta=-0.03$. (d) The traveling localized state in the presence of the nonlocal delayed response $\gamma=-0.4, \alpha=-0.1, \mu=1$, and $\eta=-0.03$. All profiles are obtained from the numerical integration of Eq. (5). The insets in (b)-(d) account for $t-\tau$ maps. When used, $D=1$.

on the sign of $\eta$. In the absence of nonlocal delay traveling LS are excluded. However, when taking into account the delayed nonlocal response, moving LS persists for the longtime evolution. An example of such behavior is shown in Fig. 2(d). The homogeneous equilibria of Eq. (5), $u_{0}$, satisfies $\eta=-(\mu+\gamma) u_{0}+u_{0}^{3}$. These solutions are altered by the strength of time-delayed nonlocal response, i.e., $\gamma$. Let us consider the superposition of two well-separated fronts as

$$
u=u_{+}(\tau-\Lambda+d / 2)+u_{-}(\tau-\Lambda-d / 2)-\sqrt{\mu}+w,
$$

where $d=d(t)$ and $\Lambda=\Lambda(t)$ account for the width and centroid between well-separated fronts $[d(t) \sqrt{\mu} \gg 1]$, as indicated in Fig. 2(d). We add to the superposition of the two fronts a small perturbation $w=w(\tau, \Lambda, d)$ with $w \ll 1$. Replacing the ansatz (6) in Eq. (5), linearizing in $w$, and imposing the solvability condition after straightforward calculations, we obtain

$$
\begin{gathered}
\dot{d}=\eta_{0}-C_{1} e^{-\sqrt{2 \mu} d}+C_{2} e^{-\alpha d}, \\
\dot{\Lambda}=C,
\end{gathered}
$$

where

$$
\begin{aligned}
\eta_{0} & \equiv \frac{4 \sqrt{\mu}}{\|\psi\|^{2}} \eta, \quad \text { with }\|\psi\|^{2} \equiv \int_{-\infty}^{\infty}\left(\partial_{\tau} u_{+}-\partial_{\tau} u_{-}\right)^{2} d \tau, \\
C_{1} & \equiv \frac{6 \mu \int_{-\infty}^{\infty} e^{-\sqrt{2 \mu} \tau} \partial_{\tau} u_{+} d \tau}{\|\psi\|^{2}}, \\
C_{2} & \equiv \frac{2 \sqrt{\mu} \int_{-\infty}^{\infty} e^{-\sqrt{|\alpha|}|\tau|} \partial_{\tau} u_{+} d \tau}{\|\psi\|^{2}} \gamma, \\
C & \equiv-\frac{2 \sqrt{\mu} \int_{-\infty}^{\infty} e^{-\alpha|\tau|} \partial_{\tau} u_{+} d \tau}{\int_{-\infty}^{\infty}\left(\partial_{\tau} u_{+}+\partial_{\tau} u_{-}\right)^{2} d \tau} \gamma .
\end{aligned}
$$

$v=\dot{d}$ and $\dot{\Lambda}$ denote the speed and the time evolution of the width, respectively. Equations (7) and (8) describe the 
(a)

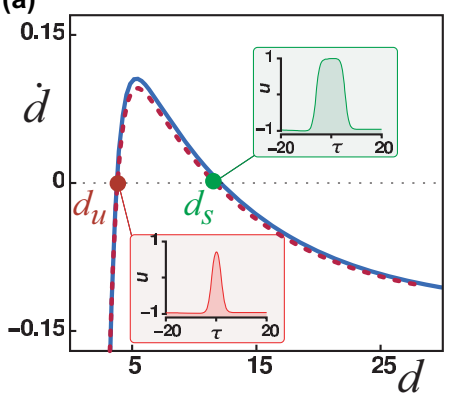

(b)

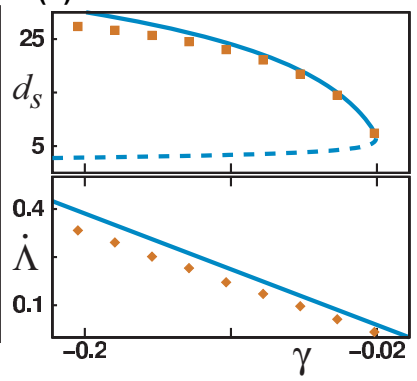

(c)

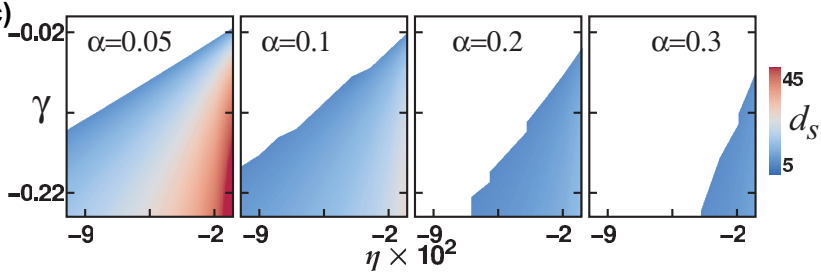

FIG. 3. Fronts interaction. (a) Interaction force $\dot{d}$ obtained by plotting Eq. (7) as a function of the width $d$ (solid curve). The dotted curve obtained from numerical simulations of Eq. (5). Both curves are obtained for $\eta=-0.03, \mu=1.00, \gamma=-0.10$, and $\alpha=0.10$. The top and bottom insets correspond to the stable $d_{s}$ and unstable $d_{u}$ traveling localized state. (b) The width $d_{s}$ (top) and speed $\dot{\Lambda}$ (bottom) of the traveling localized structure as a function of the strength of delayed nonlocal response $\gamma$. The continuous (dashed) curve corresponds to stable (unstable) traveling localized structure obtained analytically from Eqs. (7) and (8). Dot points are obtained from numerical simulations of Eq. (5). (c) Stability domain of traveling localized structures in the plane $(\eta, \gamma)$ for different values of the correlation time $\alpha$. The color map corresponds to traveling localized structures width. When used, $D=1$.

interaction between fronts. The term $\eta_{0}$, proportional to $\eta$, describes the propagation of fronts due to the potential difference between the uniform states. The term proportional to $C_{1}>0$ describes the interaction between fronts originating from the superposition of their tails. This contribution to the fronts interaction is always attractive. The time-delayed response adds a new contribution proportional to $C_{2}$ to the interaction between fronts. This term, however, can be either attractive $(\gamma<0)$ or repulsive $(\gamma>0)$. Equation (7) possesses two equilibria. The first one, $d_{u}$, corresponds to traveling LS with a small width and is always unstable. The other equilibrium with larger width, $d_{s}$, is stable as shown in Fig. 3(a). The profiles of traveling LS solutions are depicted in the insets of this figure. From dynamical system theory, these traveling LS appear thanks to a saddle-node bifurcation as shown in Fig. 3(b). They are asymmetric solutions of Eq. (5) [see also Fig. 2(d)]. This asymmetry is contained in the adjustment function $w$, and it takes source from the nonlocal delayed response. Figure 3(c) shows the stability domain of traveling LS in the plane $(\eta, \gamma)$ by varying the characteristic correlation time associated with the delayed nonlocal response. The analytical results are compared with numerical simulations of Eq. (5). This comparison involves characteristics of traveling LS such as the width $(d)$ and the speed $(\dot{\Lambda})$ as a function of the strength of nonlocal delay, $\mu$ and $\eta$. In good agreement with the analytical predictions,
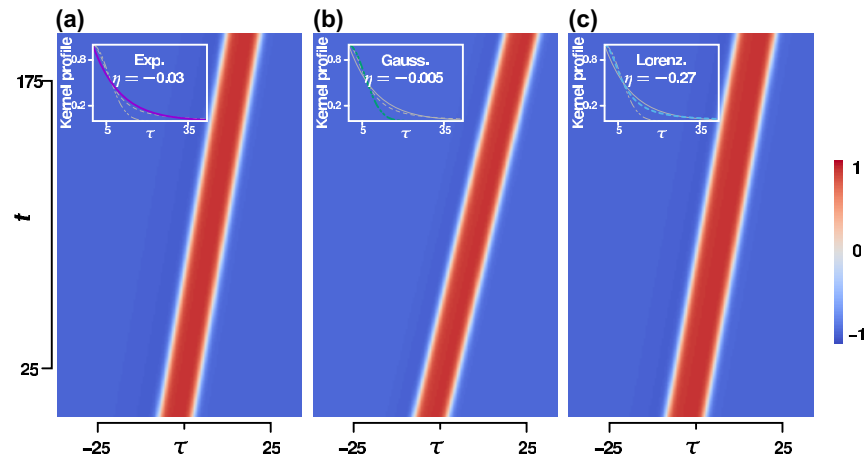

FIG. 4. Moving localized states of Eq. (3) with $\mu=1.000, \gamma=$ $-0.100, D=1$, and $\alpha=0.100$ for different types of kernel: (a) exponential $\chi(\tau)=e^{-\alpha \tau} / \alpha$ and $\eta=-0.03$, (b) Gaussian $\chi(\tau)=\alpha_{g} e^{-\left(\alpha_{g} \tau\right)^{2}} / \sqrt{\pi}$ and $\eta=-0.005$, and (c) Lorentzian $\chi(\tau)=$ $\alpha_{l} / \pi\left(1+\alpha_{l}^{2} \tau^{2}\right)$ and $\eta=-0.27$ with $\alpha_{g} \equiv \alpha / \sqrt{\ln 2}$ and $\alpha_{l} \equiv$ $\alpha / \ln 2$.

the width of traveling LS increases with the strength of all parameter values except for $\eta$.

The stabilization mechanism of traveling LS is attributed to nonlocal delayed response in the form of exponential. In addition, the same mechanism is applied to other types of kernels such as Gaussian and Lorentzian. Indeed, three kernels of the same width are considered: exponential $\chi(\tau)=e^{-\alpha \tau} / \alpha$, the Gaussian $\chi(\tau)=\alpha_{g} e^{-\alpha_{g} \tau^{2}} / \sqrt{\pi}$, and the Lorentzian $\chi(\tau)=$ $\alpha_{l} / \pi\left(1+\alpha_{l}^{2} \tau^{2}\right)$, with $\alpha_{g} \equiv \alpha / \sqrt{\ln 2}$ and $\alpha_{l} \equiv \alpha / \ln 2$. Figure 4 shows the moving localized structures obtained using exponential, Gaussian, and Lorentzian kernels. Numerical simulations are performed using a finite-difference with fourthorder Runge-Kutta scheme. The periodic boundary conditions are also used to generate moving localized structures. The formation of moving localized structures is robust with the change of the kernel. However, the kernel can change the stability domain of moving localized structures.

\section{CONCLUSIONS}

We have shown analytically and numerically that nonlocal delayed response in the form of the Raman effect stabilizes traveling localized structures. We have considered a realistic model describing all-fiber ring cavities where the nonlocal delayed coupling corresponds to the noninstantaneous Raman response. Close to the nascent bistability regime, we have derived a simple generic bistable model with a nonlocal delayed response. We have characterized these solutions by computing their width and speed. Without a nonlocal response, traveling localized solutions are excluded. Numerical solutions of the governing equations are in close agreement with analytical predictions. Finally, we have shown that the stabilization mechanism reported here holds for other types of kernels such as Gaussian and Lorentzian. These findings strongly contrast with those of previous studies and open up new possibilities for the observation of traveling localized structures in practical systems.

\section{ACKNOWLEDGMENTS}

The authors acknowledge A. Mussot for fruitful discussions. We are also grateful to the anonymous referees whose 
suggestions have improved the paper significantly. This research was funded by the Millennium Institute for Research in Optics (MIRO) and FONDECYT projects through Grant No. 1180903. M.T. received support from the Fonds National de la Recherche Scientifique (Belgium). S.C. acknowledges the LABEX CEMPI (ANR-11-LABX-0007) as well as the Ministry of Higher Education and Research, Hauts de France Council, and European Regional Development Fund (ERDF) through the Contract de Projets Etat-Region (CPER Photonics for Society $\mathrm{P} 4 \mathrm{~S})$.

\section{APPENDIX: DERIVATION OF A GENERIC BISTABLE SYSTEM FROM LUGIATO-LEFEVER EQUATION WITH RAMAN DELAYED NONLOCAL RESPONSE}

The homogeneous steady-state solutions of Eq. (1) satisfy

$$
\begin{aligned}
S= & {\left[(1+i \Delta) A_{s}-i\left(1-f_{R}\right) A_{s}\left|A_{s}\right|^{2}\right] } \\
& -i f_{R} a A_{s}\left|A_{s}\right|^{2} \int_{-\infty}^{T} H\left(T-T^{\prime}\right) d T^{\prime},
\end{aligned}
$$

with

$$
\begin{aligned}
a & =\frac{\tau_{0}\left(\tau_{1}^{2}+\tau_{2}^{2}\right)}{\tau_{1} \tau_{2}^{2}}, \quad \text { and } \\
H\left(T-T^{\prime}\right) & =e^{-\frac{\tau_{0}\left(T-T^{\prime}\right)}{\tau_{2}}} \sin \left(\tau_{0} \frac{T-T^{\prime}}{\tau_{1}}\right) .
\end{aligned}
$$

The integral appearing in the last term of Eq. (A1) has a simple expression given by

$$
\int_{-\infty}^{T} e^{-\frac{\tau_{0}\left(T-T^{\prime}\right)}{\tau_{2}}} \sin \left(\tau_{0} \frac{T-T^{\prime}}{\tau_{1}}\right) d T^{\prime}=\frac{\tau_{1} \tau_{2}^{2}}{\tau_{0}\left(\tau_{1}^{2}+\tau_{2}^{2}\right)}=\frac{1}{a} .
$$

The parameter $a$ is a normalized coefficient of the kernel $h_{R}^{\prime}(\tau)$. Note that this integral does not depend on the value of $T$. When replacing the value of this integral in Eq. (A1), we recover the well-known homogeneous steady state of the LLE [17]:

$$
S=\left[1+i\left(\Delta-\left|A_{s}\right|^{2}\right)\right] A_{s} .
$$

The homogeneous steady states remain unaltered by the Raman effect and are independent of the parameter $T$ and the strength $f_{R}$ of the Raman nonlocal effect. Let us decompose the electric field into its real and imaginary parts as $A=$ $A_{r}+i A_{i}$. By replacing this relation in Eq. (A4), we obtain

$$
\begin{gathered}
S-A_{r s}+\Delta A_{i s}-A_{i s}\left(A_{r s}^{2}+A_{i s}^{2}\right)=0, \\
-A_{i s}-\Delta A_{r s}+A_{r s}\left(A_{r s}^{2}+A_{i s}^{2}\right)=0 .
\end{gathered}
$$

In terms of intensities, the homogeneous steady-state solutions satisfy

$$
S^{2}=\left|A_{s}\right|^{2}\left[1+\left(\Delta-\left|A_{s}\right|^{2}\right)^{2}\right] .
$$

There exists a critical point marking the onset of a hysteresis loop when $\partial S / \partial\left|A_{s}\right|=\partial^{2} S / \partial\left|A_{s}\right|^{2}=0$. This corresponds to the inflection point of the intensity response curve corresponding to the critical detuning for the onset $\Delta=\Delta_{c}=\sqrt{3}$. The coordinates of the critical point are

$$
\left|A_{c}\right|^{2}=\frac{2 \sqrt{3}}{3}, \text { and } S_{c}^{2}=\frac{8 \sqrt{3}}{9} .
$$

From Eq. (A4), the intracavity field is

$A_{c}=A_{r c}+i A_{i c}, \quad$ with $\quad A_{r c}=\frac{3 S_{c}}{4} \quad$ and $\quad A_{i c}=-\frac{i \sqrt{3} S_{c}}{4}$

The bistability condition $\Delta=\Delta_{c}=\sqrt{3}$ does not depend on the Raman parameters. For $\Delta<\sqrt{3}(\Delta>\sqrt{3})$ the transmitted intensity $\left|A_{s}\right|^{2}$ as a function of the input intensity $S^{2}$ is monostable (bistable). To determine the behavior of the homogeneous steady states close to criticality, we consider the following expansion by defining a small parameter $\epsilon$ which measures the distance from the critical point as

$$
\Delta=\Delta_{c}\left(1+\delta_{1} \epsilon+\delta \epsilon^{2}\right) .
$$

We then expand also the input field amplitude, the real and the imaginary parts of the electric field in terms of $\epsilon$, as

$$
\begin{gathered}
S=S_{c}\left(1+s_{1} \epsilon+s_{2} \epsilon^{2}+s \epsilon^{3}\right), \\
A_{r s}=A_{r c}\left(1+\epsilon x_{0}+\epsilon^{2} x_{1}+\epsilon^{3} x_{2}+\cdots\right), \\
A_{i s}=A_{i c}\left(1+\epsilon y_{0}+\epsilon^{2} y_{1}+\epsilon^{3} y_{2}+\cdots\right) .
\end{gathered}
$$

By inserting expansions Eqs. (A10)-(A13) into Eqs. (A5) and (A6), we then equate the terms with the same powers of $\epsilon$. At the first order in $\epsilon$, we get $s_{1}=0, \delta_{1}=0$, and $y_{0}=-x_{0}$. At order $\epsilon^{2}$, we get

$$
s_{2}=\frac{3 \delta}{4}, \quad \text { and } \quad x_{1}=-y_{1}+\frac{3 \delta}{2}-2 x_{0}^{2} .
$$

At order $\epsilon^{3}$, we get $x_{0}^{3}-3 \delta x_{0} / 2-2 s=0$. When replacing $s_{1}=0, s_{2}=3 \delta / 4$ and $\delta_{1}=0$, we obtain

$$
\begin{gathered}
\Delta=\Delta_{c}\left(1+\delta \epsilon^{2}\right), \\
S=S_{c}\left(1+\frac{3}{4} \delta \epsilon^{2}+s \epsilon^{3}\right) .
\end{gathered}
$$

To describe the nonlinear evolution of the LLE equation in the vicinity of the critical point associated with bistability, we first decompose the electric field into its real and imaginary parts as $A=A_{r}+i A_{i}$. The evolution equations for $A_{r}$ and $A_{i}$ are

$$
\begin{aligned}
\frac{\partial A_{r}}{\partial \zeta}= & S-A_{r}+\Delta A_{i}-\left(1-f_{R}\right) A_{i}\left(A_{r}^{2}+A_{i}^{2}\right)-b_{2} \frac{\partial^{2} A_{i}}{\partial T^{2}} \\
& -f_{R} A_{i} \int_{-\infty}^{T} h_{R}^{\prime}\left(T-T^{\prime}\right) I\left(T^{\prime}\right) d T^{\prime}, \\
\frac{\partial A_{i}}{\partial \zeta}= & -A_{i}-\Delta A_{r}+\left(1-f_{R}\right) A_{r}\left(A_{r}^{2}+A_{i}^{2}\right)+b_{2} \frac{\partial^{2} A_{r}}{\partial T^{2}} \\
& +f_{R} A_{r} \int_{-\infty}^{T} h_{R}^{\prime}\left(T-T^{\prime}\right) I\left(T^{\prime}\right) d T^{\prime},
\end{aligned}
$$

with $I\left(T^{\prime}\right)=A_{r}\left(T^{\prime}\right)^{2}+A_{i}\left(T^{\prime}\right)^{2}$. We then introduce the excess variables $u$ and $v$ with respect to criticality as $A_{r s}=$ $A_{r c}(1+\epsilon u)$ and $A_{i s}=A_{i c}(1+\epsilon v)$. Replacing these relations 
in Eqs. (A17) and (A18), and by assuming that the strength of the delayed Raman effect is small, i.e., $f_{R}=f \epsilon^{2}$, we obtain

$$
\begin{aligned}
\frac{\partial u}{\partial \zeta}= & \left(\frac{v^{2}}{2}+u v+\frac{u^{2}}{2}\right) \epsilon \\
& +\left(-f u-\frac{f v}{3}-\delta v+\frac{v^{3}}{6}+\frac{v u^{2}}{2}+\frac{4 s}{3}-\frac{b_{2}}{\sqrt{3}} \frac{\partial^{2} v}{\partial T^{2}}\right) \epsilon^{2} \\
& +a f \epsilon^{2} \int_{-\infty}^{T} H\left(T^{\prime}-T\right)\left[u\left(T^{\prime}\right)+\frac{v\left(T^{\prime}\right)}{3}\right] d T^{\prime}, \quad(\mathrm{A} 19) \\
\frac{\partial v}{\partial \zeta}= & -2 u-2 v+\left(3 \delta-\frac{9 u^{2}}{2}-u v-\frac{v^{2}}{2}\right) \epsilon \\
& +\left(f v+3 f u+3 \delta u-\frac{u v^{2}}{2}-\frac{3 u^{3}}{2}+\sqrt{3} b_{2} \frac{\partial^{2} u}{\partial T^{2}}\right) \epsilon^{2} \\
& -3 f \epsilon^{2} \int_{-\infty}^{T} H\left(T^{\prime}-T\right)\left[u\left(T^{\prime}\right)+\frac{v\left(T^{\prime}\right)}{3}\right] d T^{\prime}
\end{aligned}
$$

with $H\left(T^{\prime}-T\right)=e^{-\frac{\tau_{0}\left(T^{\prime}-T\right)}{\tau_{2}}} \sin \left(\tau_{0} \frac{T-T^{\prime}}{\tau_{1}}\right)$. We seek corrections to the steady states at criticality that depends on time and space through the slow variables $t=\epsilon^{2} \zeta$ and $\tau=3^{1 / 4} \epsilon T$. We expand the variables $u$ and $v$ as

$$
\begin{aligned}
& u=u_{0}+\epsilon u_{1}+\epsilon^{2} u_{2}+\cdots, \\
& v=v_{0}+\epsilon v_{1}+\epsilon^{2} v_{2}+\cdots .
\end{aligned}
$$

Replacing the above expansions in Eqs. (A19) and (A20), we obtain at leading order in $\epsilon$,

$$
v_{0}=-u_{0} \text {. }
$$

At the next order, we obtain the following from Eq. (A20): $u_{0}^{2} / 2+u_{0} v_{0}+v_{0}^{2} / 2=0$. This relation is automatically satisfied with the previous result, i.e., $v_{0}=-u_{0}$. Finally, for a higher-order inhomogeneous problem, we obtain

$$
\begin{aligned}
\frac{\partial u_{0}}{\partial t}= & \frac{4 s}{3}+\left(\delta-\frac{2 f}{3}\right) u_{0}-\frac{2}{3} u_{0}^{3}+b_{2} \frac{\partial^{2} u_{0}}{\partial \tau^{2}} \\
& +\frac{2 f a}{3} \int_{-\infty}^{\tau} e^{-\frac{\tau_{0}\left(\tau-\tau^{\prime}\right)}{\tau_{2}}} u_{0}\left(\tau^{\prime}\right) d \tau^{\prime},
\end{aligned}
$$

and with the following changes, $t \rightarrow 2 t / 3, \eta=$ $2 s, \mu=(3 \delta-2 f) / 2, D=3 b_{2} / 2, \quad$ and $\quad \chi(\tau)=2 f a e^{-\frac{\tau_{0} \tau}{\tau_{2}}}$ $\sin \left(\tau_{0} \tau / \tau_{1}\right) / 3$, we finally obtain

$$
\partial_{t} u=\eta+\mu u-u^{3}+D \partial_{\tau \tau} u+\int_{-\infty}^{\tau} \chi\left(\tau-\tau^{\prime}\right) u\left(\tau^{\prime}\right) d \tau^{\prime} .
$$

By assuming that the function $h_{R}^{\prime}(T)$ evolves without oscillation, which corresponds to using the limits of large $\tau_{1}$ and $\tau$, i.e., we neglect $\sin \left(\tau / \tau_{1}\right)$, we obtain the generic bistable model [Eq. (5)],

$$
\partial_{t} u=\eta+\mu u-u^{3}+D \partial_{\tau \tau} u+\frac{\gamma}{\alpha} \int_{-\infty}^{\tau} e^{\alpha\left(\tau-\tau^{\prime}\right)} u\left(\tau^{\prime}\right) d \tau^{\prime},
$$

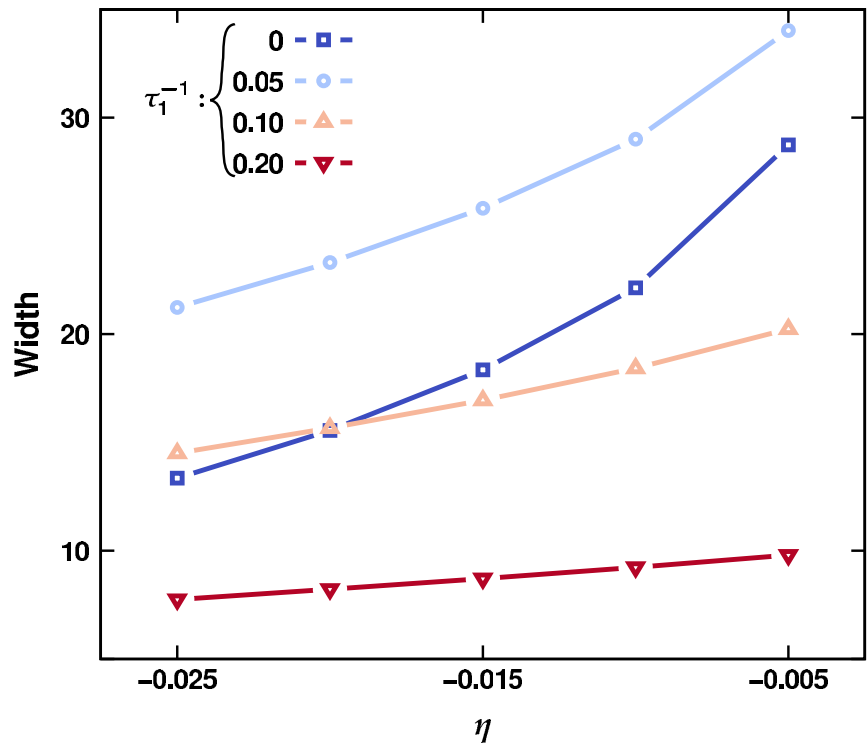

FIG. 5. Comparison between the width of moving localized structures obtained from Eqs. (A26) and (A25). Squares indicate the width obtained from Eq. (A26), and other symbols indicate the width of moving localized structures of Eq. (A25). The parameters are $\tau_{0}=5 \mathrm{fs}, \tau_{2}=32.2 \mathrm{fs}\left(\alpha=\tau_{0} / \tau_{2}=0.16\right), \gamma=.18, \mu=1.13$, and $D=1$.

where $\alpha=\tau_{0} / \tau_{2}$ and $\gamma=2 \tau_{0} f /\left(3 \tau_{2}\right)$. In order to compare both reduced equations (A25) and (A26), we performed numerical simulations of both models. The results are summarized in Figs. 5 and 6. From these figures we can see that the width, as well as the speed, of moving are similar for large $\tau_{1}$. Therefore, in this limit, both types of kernel have the same behavior.

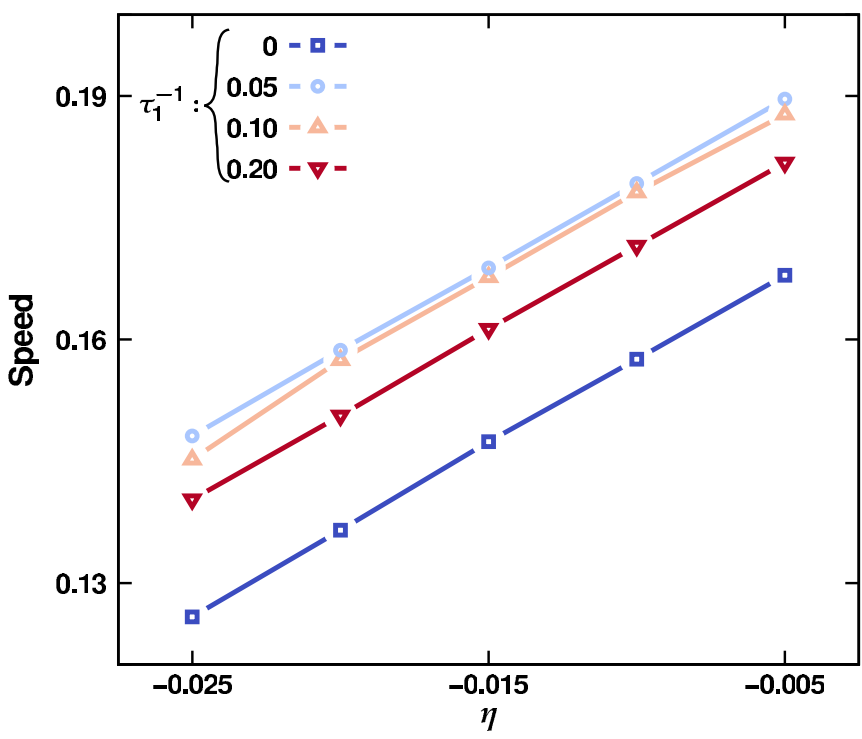

FIG. 6. Comparison between the speed of moving localized structures obtained from Eqs. (A26) and (A25). Squares indicate the speed obtained from Eq. (A26), and other symbols indicate the speed of moving localized structures of Eq. (A25). The parameters are $\tau_{0}=5 \mathrm{fs}, \tau_{2}=32.2 \mathrm{fs},\left(\alpha=\tau_{0} / \tau_{2}=0.16\right), \gamma=.18, \mu=1.13$, and $D=1$. 
[1] P. Ehrenfest and T. Ehrenfest-Afanasyeva, The Conceptual Foundations of the Statistical Approach in Mechanics, Enziklopädie der Mathematischen Wissenschaften Vol. 4. (B.G. Teubner Verlag, Leipzig, 1911).

[2] Model Reduction and Coarse-Graining Approaches for Multiscale Phenomena, edited by A. N. Gorban, N. K. Kazantzis, I. G. Kevrekidis, H. C. Ottinger, and C. Theodoropoulos (Springer-Verlag, Berlin, 2006).

[3] G. A. Voth, Coarse-Graining of Condensed Phase and Biomolecular Systems (CRC Press, Boca Raton, FL, 2008).

[4] L. D. Landau and E. M. Lifshitz, Course of Theoretical Physics, Electrodynamics of Continuous Media (Oxford University Press, Oxford, UK, 1960), Vol. 8.

[5] N. Bloembergen, Nonlinear Optics (World Scientific, Singapore, 1996).

[6] G. P. Agrawal, Nonlinear Fiber Optics (Springer, Berlin, 2000).

[7] A. Hutt, Phys. Rev. E 70, 052902 (2004).

[8] S. Coombes, Biol. Cybern. 93, 91 (2005).

[9] F. M. Atay and A. Hutt, SIAM J. Appl. Dyn. Syst. 5, 670 (2006).

[10] J. D. Murray, Mathematical Biology (Springer-Verlag, New York, 2001).

[11] P. Del'Haye, A. Schliesser, O. Arcizet, T. Wilken, R. Holzwarth, and T. J. Kippenberg, Nature (London) 450, 1214 (2007).

[12] A. J. Scroggie, W. J. Firth, G. S. McDonald, M. Tlidi, R. Lefever, and L. A. Lugiato, Chaos, Solitons Fractals 4, 1323 (1994).

[13] F. Leo, S. Coen, P. Kockaert, S. P. Gorza, P. Emplit, and M. Haelterman, Nat. Photonics 4, 471 (2010).

[14] L. A. Lugiato, F. Prati, M. L. Gorodetsky, and T. J. Kippenberg, Philos. Trans. R. Soc. London, Ser. A 376, 20180113 (2018).

[15] M. Tlidi, M. Clerc, and K. Panajotov, Philos. Trans. R. Soc. London, Ser. A, 376, 20180114 (2018).

[16] A. V. Cherenkov, N. M. Kondratiev, V. E. Lobanov, A. E. Shitikov, D. V. Skryabin, and M. L. Gorodetsky, Opt. Express 25, 31148 (2017).

[17] L. A. Lugiato and R. Lefever, Phys. Rev. Lett. 58, 2209 (1987).

[18] Y. K. Chembo, I. S. Grudinin, and N. Yu, Phys. Rev. A 92, 043818 (2015).

[19] K. J. Blow and D. Wood, IEEE J. Quantum Electron. 25, 2665 (1989).

[20] Q. Lin and G. P. Agrawal, Opt. Lett. 31, 3086 (2006).

[21] L. A. Lugiato, F. Prati, and M. Brambilla, Nonlinear Optical Systems (Cambridge University Press, Cambridge, UK, 2015).

[22] Y. K. Chembo, D. Gomila, M. Tlidi, and C. R. Menyuk, Eur. Phys. J. D 71, 299 (2017).

[23] M. Tlidi, K. Staliunas, K. Panajotov, A. G. Vladimirov, and M. G. Clerc, Philos. Trans. R. Soc. London, Ser. A 372, 20140101 (2014).

[24] S. Coen, M. Tlidi, P. Emplit, and M. Haelterman, Phys. Rev. Lett. 83, 2328 (1999).

[25] P. Parra-Rivas, E. Knobloch, D. Gomila, and L. Gelens, Phys. Rev. A 93, 063839 (2016).

[26] M. Tlidi, L. Bahloul, L. Cherbi, A. Hariz, and S. Coulibaly, Phys. Rev. A 88, 035802 (2013).

[27] A. G. Vladimirov, S. V. Gurevich, and M. Tlidi, Phys. Rev. A 97, 013816 (2018).
[28] A. Hariz, L. Bahloul, L. Cherbi, K. Panajotov, M. Clerc, M. A. Ferré, B. Kostet, E. Averlant, and M. Tlidi, Phys. Rev. A 100, 023816 (2019).

[29] Z. Liu, M. Ouali, S. Coulibaly, M. G. Clerc, M. Taki, and M. Tlidi, Opt. Lett. 42, 1063 (2017).

[30] S. Coulibaly, M. Taki, A. Bendahmane, G. Millot, B. Kibler, and M. G. Clerc, Phys. Rev. X 9, 011054 (2019).

[31] K. Panajotov, M. G. Clerc, and M. Tlidi, Eur. Phys. J. D 71, 176 (2017).

[32] K. Panajotov and M. Tlidi, Chaos 27, 013119 (2017).

[33] W. J. Firth and A. J. Scroggie, Phys. Rev. Lett. 76, 1623 (1996).

[34] L. Spinelli, G. Tissoni, M. Brambilla, F. Prati, and L. A. Lugiato, Phys. Rev. A 58, 2542 (1998).

[35] T. Maggipinto, M. Brambilla, G. K. Harkness, and W. J. Firth, Phys. Rev. E 62, 8726 (2000).

[36] E. Caboche, F. Pedaci, P. Genevet, S. Barland, M. Giudici, J. Tredicce, G. Tissoni, and L. A. Lugiato, Phys. Rev. Lett. 102, 163901 (2009).

[37] E. Caboche, S. Barland, M. Giudici, J. Tredicce, G. Tissoni, and L. A. Lugiato, Phys. Rev. A 80, 053814 (2009).

[38] A. J. Scroggie, J. M. McSloy, and W. J. Firth, Phys. Rev. E 66, 036607 (2002).

[39] L. Spinelli, G. Tissoni, L. A. Lugiato, and M. Brambilla, Phys. Rev. A 66, 023817 (2002).

[40] G. Tissoni, L. Spinelli, L. A. Lugiato, M. Brambilla, I. M. Perrini, and T. Maggipinto, Opt. Express 10, 1009 (2002)

[41] M. Santagiustina, P. Colet, M. San Miguel, and D. Walgraef, Phys. Rev. Lett. 79, 3633 (1997).

[42] P. Coullet, J. Lega, B. Houchmandzadeh, and J. Lajzerowicz, Phys. Rev. Lett. 65, 1352 (1990).

[43] D. Michaelis, U. Peschel, F. Lederer, D. V. Skryabin, and W. J. Firth, Phys. Rev. E 63, 066602 (2001).

[44] K. Staliunas and V. J. Sanchez-Morcillo, Phys. Rev. E 72 016203 (2005).

[45] P. V. Paulau, D. Gomila, T. Ackemann, N. A. Loiko, and W. J. Firth, Phys. Rev. E 78, 016212 (2008).

[46] A. J. Scroggie, W. J. Firth, and G.-L. Oppo, Phys. Rev. A 80, 013829 (2009).

[47] M. Tlidi, A. G. Vladimirov, D. Pieroux, and D. Turaev, Phys. Rev. Lett. 103, 103904 (2009).

[48] K. Panajotov and M. Tlidi, Eur. Phys. J. D 59, 67 (2010).

[49] M. Tlidi, E. Averlant, A. Vladimirov, and K. Panajotov, Phys. Rev. A 86, 033822 (2012).

[50] K. Panajotov, D. Puzyrev, A. G. Vladimirov, S. V. Gurevich, and M. Tlidi, Phys. Rev. A 93, 043835 (2016).

[51] F. Prati, G. Tissoni, L. A. Lugiato, K. M. Aghdami, and M. Brambilla, Eur. Phys. J. D 59, 73 (2010).

[52] S. V. Fedorov, A. G. Vladimirov, G. V. Khodova, and N. N. Rosanov, Phys. Rev. E 61, 5814 (2000).

[53] F. Prati, L. A. Lugiato, G. Tissoni, and M. Brambilla, Phys. Rev. A 84, 053852 (2011).

[54] S. V. Fedorov, N. N. Rosanov, and A. N. Shatsev, Opt. Spectrosc. 102, 449 (2007).

[55] N. N. Rosanov, S. V. Fedorov, and A. N. Shatsev, in Dissipative Solitons: From Optics to Biology and Medicine, edited by N. Akhmediev and A. Ankiewicz, in Lecture Notes in Physics Vol. 751 (Springer-Verlag, Berlin, 2008).

[56] V. Odent, M. Tlidi, M. G. Clerc, P. Glorieux, and E. Louvergneaux, Phys. Rev. A 90, 011806(R) (2014). 